\title{
Pathogenic importance of fibronectin in the superficial region of articular cartilage as a local factor for the induction of pannus extension on rheumatoid articular cartilage
}

\author{
S Shiozawa, R Yoshihara, Y Kuroki, T Fujita, K Shiozawa, S Imura
}

\begin{abstract}
To identify the local factors in cartilage that are responsible for the induction of pannus invasion, a 14 day organ culture study in which rheumatoid synovium was grown in contact with cartilage pieces was carried out. Rheumatoid synovium preferentially extended over hyaluronidase treated cartilage pieces, but detached from untreated pieces. Rheumatoid synovium extended over hyaluronidase treated cartilage surfaces containing fibronectin more extensively than over surfaces treated with hyaluronidase only. Extension over hyaluronidase treated cartilage surfaces containing immune complexes was small. The adherence of synovial cells to hyaluronidase treated cartilage slices in vitro was specifically inhibited by the synthetic peptide, GlyArg-Gly-Asp-Ser-Pro, which is the adhesive portion of the fibronectin molecule. Furthermore, synovial fibroblast-like cellular extension, morphologically similar to rheumatoid pannus, was observed in the organ culture experiments in which rheumatoid synovium grew over hyaluronidase treated cartilage surfaces containing fibronectin. Synovial tissue extension over fibronectin coated surfaces was inhibited when hyaluronic acid and chondroitin-4-sulphate, major components of cartilage proteoglycans, were present on the cartilage surface. These findings suggest that fibronectin present in the superficial region of cartilage potentiates rheumatoid synovial extension and proteoglycans and immune complexes inhibit rheumatoid synovial extension. It is likely that fibronectin deposited on the eroded surface of articular cartilage induces pannus formation in rheumatoid arthritis.
\end{abstract}

(Ann Rheum Dis 1992; 51: 869-873)

Pannus invasion begins at the margin of articular cartilage that borders the junction between synovial tissue and cartilage. Pannus destroys cartilage either by growing over its surface ${ }^{1}$ or directly penetrating the bare zone between cartilage and synovial insertion, thereby migrating into the medullary space of bone. ${ }^{2}$ On the articular surface of cartilage, pannus first extends as a morphologically quiescent layer of fibroblast-like cells. This is followed by matrix degradation by macrophage-like cells underneath this fibroblastic cell layer in the absence of blood vessels. ${ }^{13}$
Degradation of cartilage in the absence of vascular supply suggests that local factors in the cartilage matrix might stimulate pannus formation. The most likely factors are immune complexes $^{45}$ and fibronectin, ${ }^{6}$ detectable in the superficial region of rheumatoid articular cartilage in a disease specific manner.

To identify the local factors in cartilage that are responsible for the induction of pannus formation, an organ culture study allowing the interaction of rheumatoid synovium with cartilage pieces was carried out. The results obtained indicate that fibronectin may be a local factor responsible for rheumatoid pannus formation.

Patients and methods SAMPLES AND REAGENTS

Samples of rheumatoid synovium were obtained during joint operations on patients fulfilling the diagnostic criteria for classic rheumatoid arthritis. ${ }^{7}$ Cartilage specimens obtained from osteoarthritic knee joints were stored at $-20^{\circ} \mathrm{C}$. Cartilage specimens were trimmed to a flat shape, and freed of the original articular surface to remove any inequalities between the articular surface and cut surface with regard to synovial derived tissue or deposited substances, or both. They were either left untreated, or treated with $300 \mathrm{U} / \mathrm{ml}$ of bovine testicular hyaluronidase (Sigma Chemical, St Louis, MO, USA) at $37^{\circ} \mathrm{C}$ for three hours to remove proteoglycans from the cartilage according to previous results. ${ }^{6}$ One side of the cartilage specimens was treated with either immune complexes, human fibronectin (Sigma), or proteoglycans $(10 \mathrm{mg} / \mathrm{ml}$ hyaluronic acid and 3\% chondroitin-4-sulphate) at room temperature for one hour (fig 1). After washing with $0 \cdot 1 \mathrm{M}$ phosphate buffer, $\mathrm{pH} 7 \cdot 2$, small long cartilage pieces were cut out perpendicularly to the flat cartilage surface. Horseradish peroxidase-antiperoxidase $^{8}$ (Dakopatts, Glostrup, Denmark) was used as the immune complex. Histochemical staining for the detection of fibronectin on the cartilage surfaces was carried out as described previously using antibodies to human fibronectin and the diaminobenzidine hydrogen peroxide reaction, ${ }^{6}$ and immune complexes were detected by directly applying the diaminobenzidine hydrogen peroxide reaction.

The culture medium was Iscove's modified Dulbecco's minimal essential medium (Gibco Laboratories, Grand Island, NY, USA) supplemented with transferrin, soybean lecithin,

\footnotetext{
Correspondence to: Dr Shiozawa.

Accepted for publication 4 November 1991

Department of
Medicine,
Third Division,
Kobe University

School of Medicine,
7-5 Kusunokicho,

Chuoku, Kobe 650

S Shiozawa

Y Kuroki

Department of

Rheumatology,

Kakogawa National

Saijo, Kakogawa, Japan
} 
delipidated albumin, and $10 \%$ fetal calf serum (IMDM). ${ }^{9}$

ORGAN CULTURE

Rheumatoid synovium, minced with scissors, was layered in the centre of a Millipore filter (HATF 02500, Nihon Millipore Kogyo KK, Yonezawa, Japan). Rheumatoid synovium was attached to the ends of cartilage pieces. They were cultured in an organ culture dish (Falcon No 3037, Becton Dickinson, Cockeysville, MD, USA) in a humidified atmosphere of $5 \%$ $\mathrm{CO}_{2}$ at $37^{\circ} \mathrm{C}$ for 14 days (fig 1 ).

SYNOVIAL CELL CULTURE ON CARTILAGE SLICES Cartilage specimens punched out in a cylindrical shape $(4 \mathrm{~mm}$ diameter) were snap frozen.

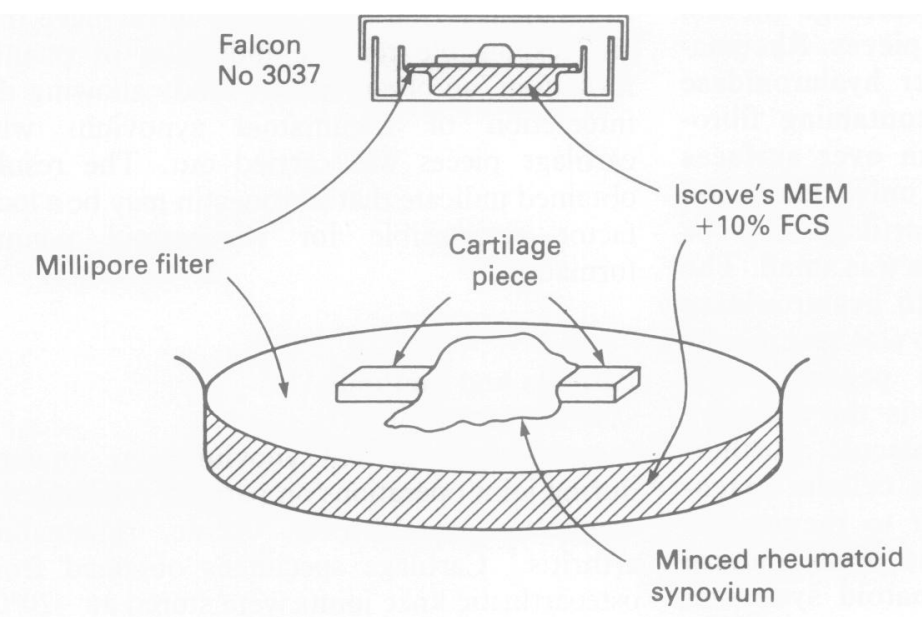

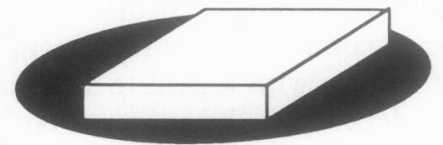

One side of the cartilage specimen is reacted with IC, Fn and/or PG

A

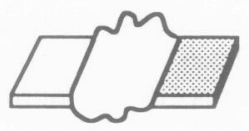

B

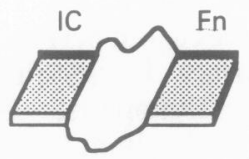

C

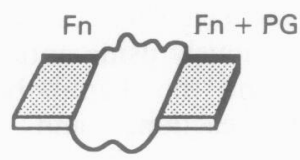

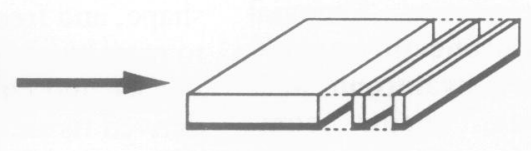

A thin cartilage slice is cut out
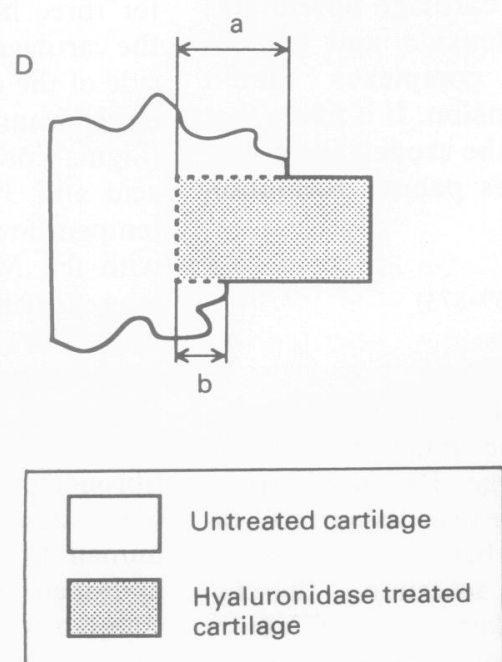

Figure 1 Organ culture experiment. Rheumatoid synovium is grown in contact with cartilage pieces on a Millipore filter at $37^{\circ} \mathrm{C}$ for 14 days. One side of the cartilage specimens is treated with immune complexes $(I C)$, fibronectin $(F n)$, and/or proteoglvcans $\left(I^{\prime}(j)\right.$. Thin cartilage slices are cut out and positioned in contact with rheumatoid synovium as shown in $A-C$. The distance of rheumatoid synovial extension is measured as shown in $D$.
Frozen cartilage $20 \mu \mathrm{m}$ thick was cut within a cryostat, attached to cover slips using $1 \%$ bovine serum albumin, and allowed to dry. The cartilage slices were then treated with $300 \mathrm{U} / \mathrm{ml}$ of hyaluronidase at $37^{\circ} \mathrm{C}$ for three hours. Synovial tissue cells were dispersed by treatment with $2 \mathrm{mg} / \mathrm{ml}$ collagenase from Clostridium histolyticum (Wako Pure Chemicals, Osaka, Japan) at $37^{\circ} \mathrm{C}$ for 45 minutes and $0.05 \%$ trypsin (Flow Laboratories, McLean, VA, USA) at $37^{\circ} \mathrm{C}$ for 30 minutes. After overnight culture, synovial adherent cells were harvested and laid on a cartilage slice $\left(2 \times 10^{2} / \mathrm{mm}^{3}\right.$ cartilage slice in $10 \mu \mathrm{l}$ IMDM). After incubation at $37^{\circ} \mathrm{C}$ for one hour they were cultured in a 24 well culture dish (No 25820, Becton Dickinson) by adding 1 $\mathrm{ml} /$ well of IMDM in the presence of $0.6 \mathrm{mM}$ fibronectin peptide (Gly-Arg-Gly-Asp-Ser-Pro) or its control peptide (Gly-Arg-Gly-Glu-SerPro) (Iwaki Glass, Tokyo, Japan) at $37^{\circ} \mathrm{C}$ for six days.

\section{MORPHOLOGICAL STUDY}

Culture was stopped by fixing specimens with $0.5 \%$ glutaraldehyde at $4^{\circ} \mathrm{C}$ for 24 hours. Tissues were then observed with a Nikon SMZ 10 stereomicroscope, or processed for dehydration in graded alcohol and embedding in Epon 812 . Sections $300 \mathrm{~nm}$ thick were cut and observed under light microscopy with toluidine blue staining. The extent of synovial tissue extension over the cartilage surfaces was measured in a stereomicroscope from the reverse side (the side attached to the Millipore filter) of the tissue by placing a scale, accurate to $0.1 \mathrm{~mm}$, underneath the tissue (fig 1D).

\section{Results}

In the organ culture experiments in which rheumatoid synovium was reacted with cartilage pieces for 14 days (fig 1A), rheumatoid synovium preferentially extended over hyaluronidase treated cartilage pieces, but detached from untreated cartilage pieces (fig 2).

To examine the part played by immune complexes and fibronectin localised in the superficial region of cartilage matrix in pannus formation, the hyaluronidase treated cartilage surfaces were covered with horseradish peroxidase-antihorseradish peroxidase immune complexes or with fibronectin (fig 1B). They were then reacted with rheumatoid synovium.

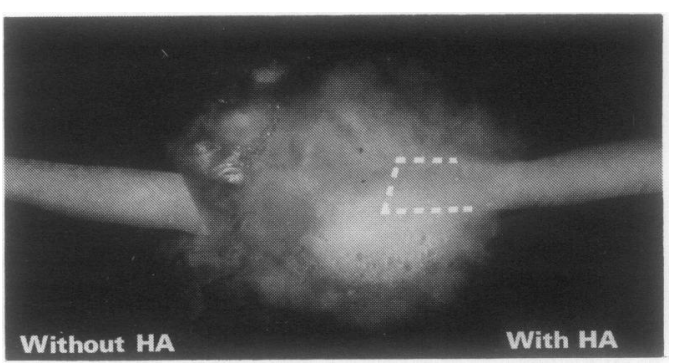

Figure 2 Rheumatoid synovium extends on the hyaluronidase $(H A)$ treated cartilage piece, but detaches from untreated cartilage after 14 days of organ culture. The original portion of the ends of the cartilage piece is indicated by dotted lines. 
After 14 days of culture, rheumatoid synovium extended poorly over the immune complex coated cartilage surfaces (table), but extended over fibronectin coated cartilage surfaces more extensively than over hyaluronidase treated cut surfaces of cartilage (table). On the cut surface of cartilage, fibronectin endogenously present in the matrix was exposed by the hyaluronidase treatment, and the amount of fibronectin on the cartilage was additionally increased by treatment with fibronectin (fig 3; see also table 1 in Shiozawa $e t a l^{6}$ ).

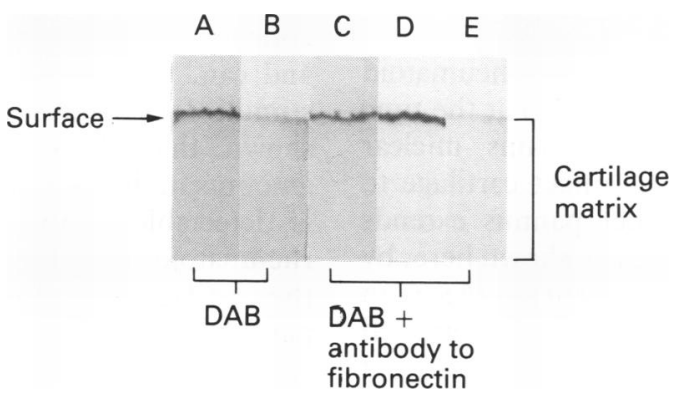

Figure 3 Cut surfaces of cartilage treated with: $(A) 300$ U/ml hyaluronidase at $37^{\circ} \mathrm{C}$ for three hours, and subsequently with horseradish peroxidase-antiperoxidase immune complexes; $(B)$ and $(C)$ with hyaluronidase alone; $(D)$ with hyaluronidase and fibronectin; or $(E)$ with hyaluronidase, fibronectin, and subsequently coated with hyaluronic acid and chondroitin-4-sulphate. Immune complexes are visualised by directly applying the diaminobenzidine hydrogen peroxide $(D A B)$ reaction in $A$ and $B$. Fibronectin is visualised by antibodies to human fibronectin and the $D A B$ reaction in $C-E$.

Rheumatoid synovial extension on hyaluronidase treated cartilage in organ culture of rheumatoid synovium with a cartilage piece

\begin{tabular}{|c|c|c|c|}
\hline & Experiment 1 & Experiment 2 & Experiment 3 \\
\hline $\begin{array}{l}\text { Fibronectin/nil } \\
\text { Immune complex/nil }\end{array}$ & $\begin{array}{l}1 \cdot 60 \dagger \\
0.40\end{array}$ & $\begin{array}{l}1 \cdot 50 \\
0 \cdot 21\end{array}$ & $\begin{array}{l}1 \cdot 38 \\
0 \cdot 12\end{array}$ \\
\hline $\begin{array}{l}\text { Fibronectin and } \\
\text { proteoglycans/nil }\end{array}$ & 0.42 & 0.01 & 0.51 \\
\hline
\end{tabular}

${ }^{*}$ Hyaluronidase treated cartilage surfaces are either left untreated (nil), coated with fibronectin (fibronectin), coated with horseradish peroxidase-antiperoxidase immune complexes (immune complex), or coated with fibronectin and then with hyaluronic acid and chondroitin-4 sulphate (fibronectin and proteoglycans).
+ Ratio of synovial extension on one side of the cartilage (a) to the other (b) in fig $1 \mathrm{D}$.
To examine further the part played by fibronectin, a dispersed adherent population of synovial cells $\left(2 \times 10^{2} / \mathrm{mm}^{3}\right)$ was cultured on a hyaluronidase treated cartilage slice in the presence of the synthetic peptide, Gly-Arg-GlyAsp-Ser-Pro, which is the adhesive portion of the fibronectin molecule that specifically inhibits binding of fibronectin to cells. ${ }^{10}$ After six days of culture, synovial cell adherence was significantly inhibited by Gly-Arg-Gly-Asp-SerPro, but not by the control peptide, Gly-ArgGly-Glu-Ser-Pro(fig 4). In the culture containing the control peptides, synovial cells adhered to and extended with long dendritic processes on the cartilage slice. In the culture containing the fibronectin peptides, the number of cells attached to the cartilage slice was less than $1 / \mathrm{mm}^{2}$ in three experiments.

Synovial fibroblast-like cellular extension, morphologically similar to rheumatoid pannus, was observed in the organ culture specimens of rheumatoid synovium with the hyaluronidase treated cartilage surfaces containing fibronectin (fig 5A-C). Some cells beginning to invade the cartilage underneath the tissue were noted (arrow). This type of mesenchymal cellular

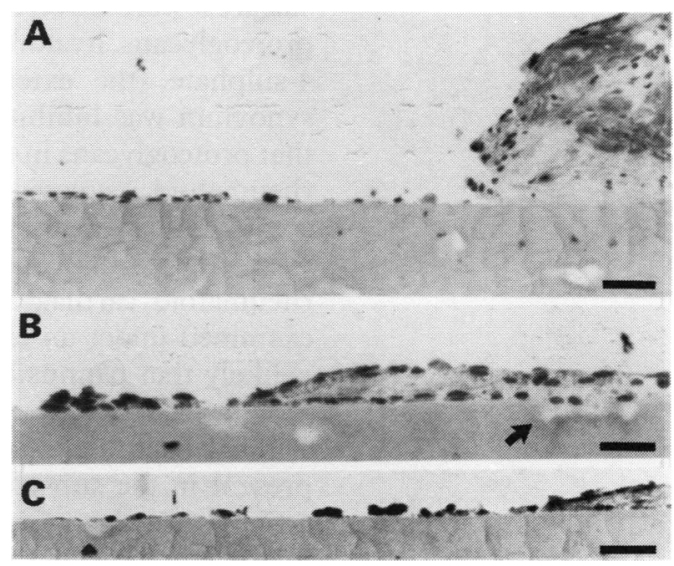

Figure 5 Rheumatoid synovium extends on the hyaluronidase treated cartilage surface containing fibronectin. A continuous tissue section is shown from $A$ to $C$ Cartilage matrix is focally degraded underneath the tissue (arrow). Bar $=50 \mu \mathrm{m}$.
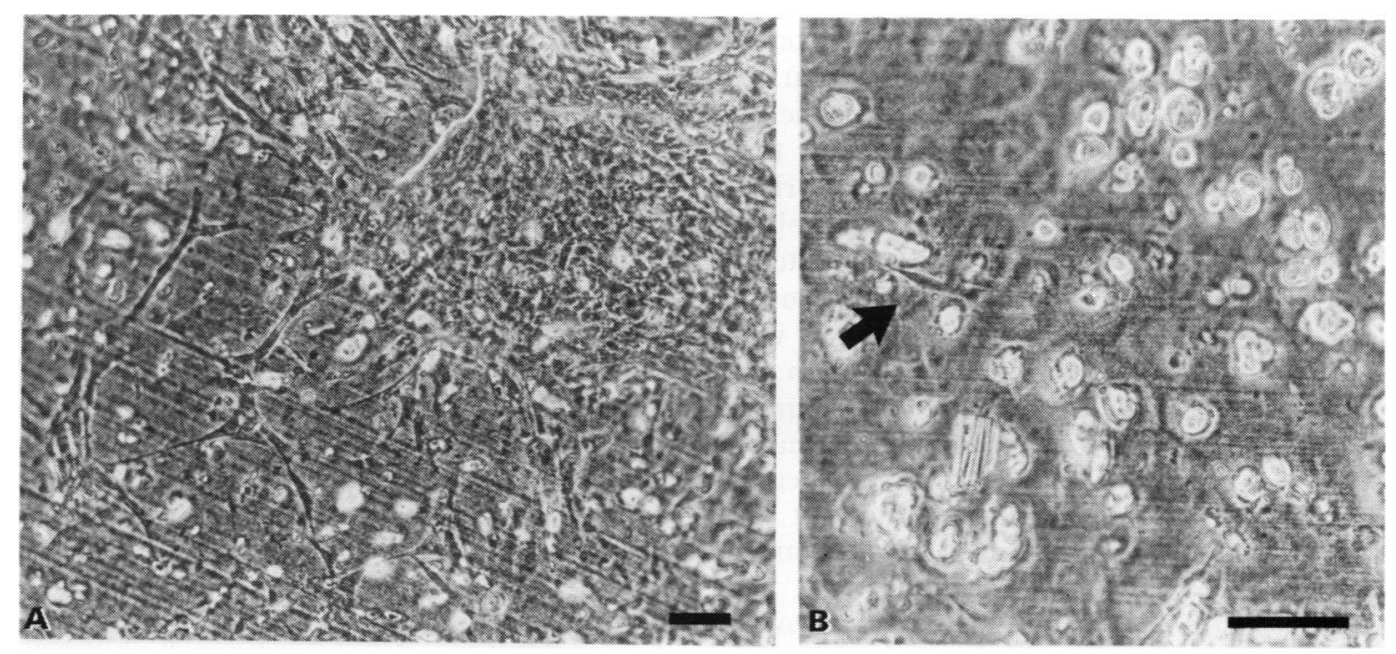

Figure 4 Synovial adherent cells $\left(2 \times 10^{2} / \mathrm{mm}^{2}\right)$ cultured on a cartilage slice for six davs in the presence of $0.6 \mathrm{mM}$ of either Gly-Arg-Gly-Glu-Ser-Pro, a control peptide (A) or Glv-Arg-(Glv-Asp-Ser-I'ro, a fibronectin peptide (B). Synovial cells extend with long dendritic processes and a cell cluster is formed on the curtilage slice in $A$, whereas onlv one cell is detectable in B. Bar $=50 \mu \mathrm{m}$. 
extension was not observed in the hyaluronidase treated cartilage surfaces containing immune complexes (not shown).

When the hyaluronidase treated cartilage surfaces containing fibronectin were additionally coated with hyaluronic acid and chondroitin-4sulphate (major components of proteoglycans), artificially reproducing the normal cartilage surface (fig 1C), synovial tissue extension was significantly inhibited (table). Adherence was greater in the presence of fibronectin, except in the presence of proteoglycans (fig $3 \mathrm{E}$ ).

\section{Discussion}

Previous studies have shown that rheumatoid cartilage surfaces are already eroded at the time of pannus invasion. ${ }^{11} 12$ It remains unclear whether pannus invades the intact cartilage to erode its surface, or whether pannus extends over an eroded surface. We have shown here, by using hyaluronidase treated cartilage pieces in which proteoglycans had been partially removed from the cartilage surface, ${ }^{6}$ that rheumatoid synovium preferentially extends on the hyaluronidase treated cartilage surfaces. Furthermore, when the hyaluronidase treated cartilage surfaces were covered by the components of proteoglycans, hyaluronic acid, and chondroitin4-sulphate, the extension of the rheumatoid synovium was inhibited. These results suggest that proteoglycans in the cartilage matrix inhibit rheumatoid synovial extension. Thus, in conjunction with the previous observation that even the normal appearing joint surfaces of rheumatoid cartilage are already eroded when examined under an electron microscope, ${ }^{12-14}$ it is likely that pannus extends only on an eroded articular cartilage.

It has been suggested that immune complexes present in the superficial region of rheumatoid articular cartilage might be an important factor in the invasion of the cartilage by pannus. ${ }^{45}$ The area covered by pannus failed to show immune complexes, however, even in the areas immediately adjacent to the advancing edge of the pannus which, presumably, had only recently been invaded by the granulation tissue. ${ }^{14}$ This suggests that immune complexes, even though they may be conveying an initial signal to pannus formation, cannot be the stimulus for the advance of the pannus. ${ }^{3}$ The present observations showing that immune complexes are poor inducers of rheumatoid synovial extension may be compatible with this. In addition, the finding is consistent with the previous observation that patients with hypogammaglobulinaemia presented with destructive arthritis far more often than expected from their small amounts of immune complexes. ${ }^{15}$ It thus appears that immune complexes sequestered in the superficial region of rheumatoid articular cartilage do not play a major part in the induction of pannus extension onto cartilage surface.

Rheumatoid synovium extended on the hyaluronidase treated cartilage surfaces containing fibronectin more extensively than on the surfaces with hyaluronidase treatment alone. The attachment of synovial cells on the cartilage matrix in vitro was inhibited by the fibronectin peptide, Gly-Arg-Gly-Asp-Ser-Pro, which specifically inhibits binding of fibronectin to cells, ${ }^{10}$ indicating that fibronectin in the cartilage matrix is required for the adherence and extension of synovial cells. Furthermore, synovial fibroblast-like cellular extension similar to rheumatoid pannus was experimentally reproduced in vitro by allowing rheumatoid synovium to grow over hyaluronidase treated cartilage surfaces containing fibronectin. These findings suggest that fibronectin, present in the superficial region of cartilage, potentiates rheumatoid synovial extension. Fibronectin is endogenously present in the cartilage matrix, ${ }^{16}$ and can, therefore, be a source of continuous stimuli for pannus formation. Studies have shown that fibronectin is present in large amounts in the rheumatoid joint, ${ }^{17-20}$ and that it is detectable in the superficial region of the rheumatoid articular cartilage. ${ }^{6}$ Fibronectin in the cartilage matrix appeared to be revealed by partial degradation of proteoglycan by proteolytic enzymes in the synovial fluid, as well as by the deposition of fibronectin on the cartilage surface. ${ }^{6}$ It thus appears that fibronectin deposited on the eroded surface of rheumatoid articular cartilage potentiates pannus invasion in vivo.

Studies using in vitro culture of synovial cells with collagenous matrix have shown that collagenase plays a central part in the promotion of joint destruction, in which the interaction between plasmin, an enhancing factor, and the tissue inhibitor of metalloproteinases, an inhibitory factor, regulates its final outcome. ${ }^{21}$ Fibronectin present in the collagenous matrix appears to promote matrix degradation by collagenase by enhancing the attachment of pannus cells to the matrix. ${ }^{3} 22$

This work was supported by the Japan Ministry of Education Science and Culture grant (S.S). We thank our teacher, Professor Morris Ziff, for useful advice.

1 Shiozawa S, Shiozawa K, Fujita T. Morphological observation in the early phase of the cartilage-pannus junction, Light in the early phase of the cartilage-pannus junction, Light and electron microscopic studies

2 Fassbender H G. Joint destruction in various arthritic diseases. In: Kuettner K, ed. Articular cartilage biochemistry. New York: Raven Press, 1986: 371-89.

3 Shiozawa S, Shiozawa K. A review of the histopathological evidence on the pathogenesis of cartilage destruction in rheumatoid arthritis. Scand $\mathcal{7}$ Rheumatol Suppl 1988; 74 65-72.

4 Cooke T D, Hurd E R, Ziff M. Identification of immunoglobulins and complement in rheumatoid articular collagenous tissues. Arthritis Rheum 1975; 18: 541-51.

5 Ishikawa H, Smiley J D, Jasin H E, Bienenstock J, Ziff $M$ Electron microscopic demonstration of immunoglobulin Electron microscopic demonstration of immunoglobulin 18: $563-76$.

6 Shiozawa K, Shiozawa S, Shimizu S, Fujita T. Fibronectin on the surface of articular cartilage in rheumatoid arthritis. Arthritis Rheum 1984; 27: 615-22

7 Arnett F C, Edworthy S M, Bloch D A, et al. The American Rheumatism Association 1987 revised criteria for the classification of rheumatoid arthritis. Arthritis Rheum 1988 31: 315-24.

8 Sternberger L A, Hardy P H Jr, Cuculis J J, Meyer H G. The unlabeled antibody enzyme method of immunohistochemistry. Preparation and properties of soluble antigenantibody complex (horseradish peroxidase-antihorseradish peroxidase) and its use in identification of spirochetes. 7 Histochem Cytochem 1970; 18: 315-33.

9 Shiozawa K, Shiozawa S, Shimizu S, Fujita T. l 1,25 dihydroxyvitamin D3 inhibits pokeweed mitogen-stimulated human B-cell activation: an analysis using serum-free culture conditions. Immunologv 1985; 56: 161-7.

10 Pierschbacher M D, Ruoslahti E. Cell attachment activity of fibronectin can be duplicated by small synthetic fragments fibronectin can be duplicated by small sy
of the molecule. Nature $1984 ; 309: 30-3$.

11 Chaplin D M. The pattern of bone and cartilage damage in 
the rheumatoid knee. 7 Bone Joint Surg /Br/ 1971; 53: 711-7

12 Kimura $H$, Tateishi $H$, Ziff $M$. Surface ultrastructure of rheumatoid articular cartilage. Arthritis Rheum 1977; 20: rheumatoid

13 Mitchell N, Shepard N. The ultrastructure of articular cartilage in rheumatoid arthritis. A preliminary report. f Bone foint Surg [Am] 1970; 52: 1405-23.

14 Shiozawa S, Jasin H E, Ziff $M$. Absence of immunoglobulins in rheumatoid cartilage-pannus junctions. Arthritis Rheum 1980; 23: 816-21.

15 Good R A, Rotstein J. Rheumatoid arthritis and agammaglobulinemia. Bull Rheum Dis 1960; 10: 203-6.

16 Weiss R E, Reddi A H. Appearance of fibronectin during the differentiation of cartilage, bone, and bone marrow. $\mathcal{J}$ Cell Biol 1981; 88: 630-6

17 Carson S, Mosesson M W, Diamond H S. Detection and quantitation of fibronectin in synovial fluid from patients quantitation of fibronectin in synovial fluid from patients
with rheumatic disease. Arthritis Rheum 1981; 24: 1261-7.
18 Vartio T; Vaheri A, von Essen $\mathrm{R}$, Isomaki $\mathrm{H}$, Stenman $\mathrm{S}$. Fibronectin in synovial fluid and tissue in rheumatoid arthritis. Eur f Clin Invest 1981; 11: 207-12.

19 Scott D L, Delamere J P, Walton K W. The distribution of fibronectin in the pannus in rheumatoid arthritis. $B r \mathcal{F}$ Exp Pathol 1981; 62: 362-7.

20 Shiozawa S, Ziff $M$. Immunoelectron microscopic demonstration of fibronectin in rheumatoid pannus and at the cartilage-pannus junction. Ann Rheum Dis 1983; 42: 254-63.

21 Reynolds J J, Lawrence C E, Gavrilovic J. Model systems for studying the destruction of the extracellular matrix and their usefulness in testing inhibitors. In: Glauert A M, ed. The control of tissue damage. Amsterdam: Elsevier, 1988: 281-96.

22 Shiozawa S, Tokuhisa T. Contribution of synovial mesenchymal cells to the pathogenesis of rheumatoid arthritis. Semin Arthritis Rheum 1992; 21: 267-73. 